\title{
Hepatitis A Virus Capsid Structure
}

\author{
David I. Stuart, ${ }^{1,2}$ Jingshan Ren, ${ }^{1}$ Xiangxi Wang, ${ }^{3}$ Zihe Rao, ${ }^{3,4}$ and Elizabeth E. Fry ${ }^{1}$ \\ ${ }^{1}$ Division of Structural Biology, The Henry Wellcome Building for Genomic Medicine, The Wellcome Trust \\ Centre for Human Genetics, University of Oxford, Oxford OX3 7BN, UK \\ ${ }^{2}$ Diamond Light Source Ltd, Diamond House, Harwell Science and Innovation Campus, Didcot OX11 ODE, UK \\ ${ }^{3}$ National Laboratory of Biomacromolecules, Institute of Biophysics, Chinese Academy of Science, Beijing \\ 100101, China \\ ${ }^{4}$ Laboratory of Structural Biology, School of Medicine, Tsinghua University, Beijing 100084, China \\ Correspondence: liz@strubi.ox.ac.uk
}

Hepatitis A virus (HAV) has been enigmatic, evading detailed structural analysis for many years. Its recently determined high-resolution structure revealed an angular surface without the indentations often characteristic of receptor-binding sites. The viral protein 1 (VP1) $\beta$ barrel shows no sign of a pocket factor and the amino terminus of VP2 displays a "domain swap" across the pentamer interface, as in a subset of mammalian picornaviruses and insect picorna-like viruses. Structure-based phylogeny confirms this placement. These differences suggest an uncoating mechanism distinct from that of enteroviruses. An empty capsid structure reveals internal differences in VP0 and the VP1 amino terminus connected with particle maturation. An HAV/Fab complex structure, in which the antigen-binding fragment (Fab) appears to act as a receptor-mimic, clarifies some historical epitope mapping data, but some remain difficult to reconcile. We still have little idea of the structural features of enveloped HAV particles.

$T_{e}^{\text {he }}$ he primary function of the picornavirus outer protein shell (capsid) is to protect the genome and evade immune recognition to enable the transport of the genome into the cellular cytoplasm where replication can be initiated. The structure of the capsid can help us to understand some aspects of this process: how capsid assembly is directed and controlled, how the genome is encapsidated, how interactions with the host cell are coordinated, how the capsid disassembles to facilitate genome replication and how the capsid organization responds to and evades the antiviral response mounted by the host.

The Picornaviridae are an extremely wellstudied family of viruses; however, the Hepato- virus genus of which hepatitis A virus (HAV) is the type species are somewhat outliers from, and much less well studied than, for instance, the enteroviruses (e.g., poliovirus and rhinoviruses). Picornaviruses generally possess a nonenveloped $\sim 30 \mathrm{~nm}$ diameter capsid that packages a single-stranded RNA genome. The genomic RNA contains a single large open reading frame, flanked on each end by relatively short nontranslated RNA segments. The open reading frame encodes a single large polyprotein that is processed by a viral protease into three polypeptide intermediates, P1-P3. P1 is subsequently further processed into three structural proteins, VP0, VP3, and VP1, which self-assemble into a

Editors: Stanley M. Lemon and Christopher Walker

Additional Perspectives on Enteric Hepatitis Viruses available at www.perspectivesinmedicine.org

Copyright (C) 2019 Cold Spring Harbor Laboratory Press; all rights reserved; doi: 10.1101/cshperspect.a031807

Cite this article as Cold Spring Harb Perspect Med 2019;9:a031807 
D.I. Stuart et al.

spherical capsid with icosahedral (532) symmetry (Fig. 1A). VP0, VP3, and VP1 are roughly similar in shape and arranged so as to approximate $T=3$ quasi-equivalence (Rossmann and Johnson 1989). In many picornaviruses, VP0 undergoes a final maturation cleavage after packaging of the RNA genome, by a poorly understood mechanism, perhaps catalyzed by RNA. This yields the mature VP2 capsid protein and a smaller amino-terminal fragment, VP4.

The general structure of the picornaviral capsid has been well characterized. The three largest capsid proteins, VP1-3, each adopt an eightstranded antiparallel " $\beta$-barrel" fold in which the amino-terminal amino sequences are positioned on the inner surface and the carboxy-terminal residues face externally. The small VP4 protein is located internally and not well visualized in crystallographic models, either because it is flexible or because it contacts the single mole-

A

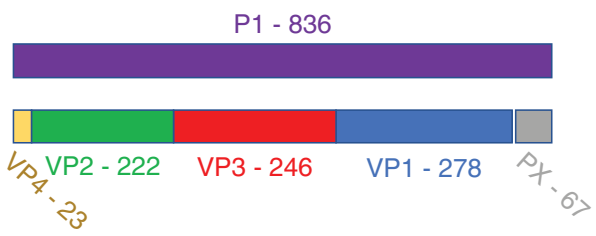

B

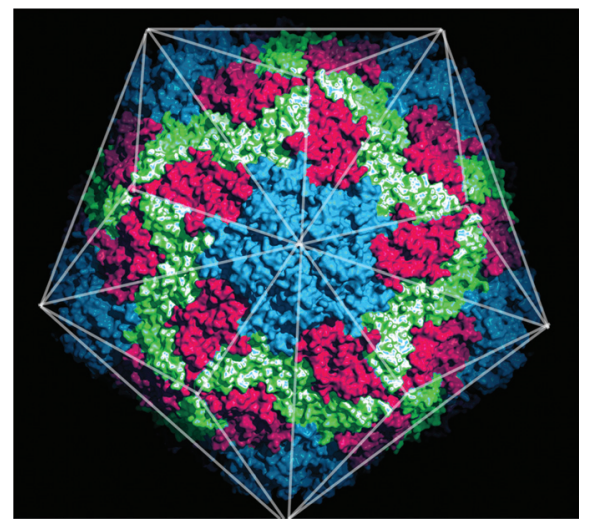

Figure 1. HAV structural proteins and their topography. (A) Schematic of polypeptide intermediate 1 (P1) genome organization. The numbers of amino acids in the polypeptides are shown. The color scheme is viral protein 1 (VP1), blue; VP2, green; VP3, red; VP4, yellow; this is used throughout the figures. $(B)$ Surface overview: the hepatitis A virus (HAV) accessible surface. White lines clarify the particle facets. cule of genomic RNA, which does not possess strict icosahedral symmetry. Five copies of the VP1 capsid protein surround the icosahedral fivefold axes. Three copies of VP2 and VP3 alternate at the threefold axes, and two copies of VP2 abut each other at the twofold axes (Fig. 1B).

Interactions of the external surface of the assembled capsid with a receptor expressed on the surface of the host cell play a critical role in cellular entry of the virus. The surface of the capsid also interacts with antibodies generated by the host's immune system, potentially resulting in neutralization of infectivity. The peptide loops on the surface of the capsid have little structural constraint, and thus tolerate deletions, insertions, or amino acid substitutions that enable the virus to evade antibodies. These surface loops therefore vary significantly in length and structure, so that different picornavirus species may have distinctive shapes. Thus, viruses of one major genus-Enterovirus - have a depression (canyon) around the fivefold vertices, which is highly conserved and frequently harbors the site of receptor interaction (Rossmann et al. 1985). Close to this is an opening into a hydrophobic pocket in the interior of the VP1 $\beta$-barrel in which a lipidic molecule (pocket factor) binds (Smith et al. 1986). In enteroviruses, it seems that the lipid is dislodged from the pocket, leading to its collapse, after binding of the capsid to its receptor and concomitant uptake into early endosomes. These conformational rearrangements in the capsid result in channels opening up both on and adjacent to the twofold axes that allow VP4 and the amino terminus of VP1, both hydrophobic and positioned internally, to externalize and puncture the endosomal membrane (Ren et al. 2013). This triggers release of the viral RNA, which enters the cytoplasm where it undergoes translation on ribosomes and initiates the process of replication (Wang et al. 2012). Other genera of the Picornaviridae do not possess the VP1-binding pocket, and the site of receptor binding can vary. It is quite possible that the mechanism of uncoating varies with the site of receptor binding, but the details are less well established.

HAV has markedly different physicochemical properties to nearly all other picornaviruses (Siegl et al. 1981). It is remarkably stable both 
genetically and physically, being able to withstand temperatures up to $80^{\circ} \mathrm{C}$ and $\mathrm{pH}$ as low as 2 , and empty particles appear even more robust than full particles (Wang et al. 2015). This makes disinfection tricky and the process of uncoating, for now, unfathomable. Quite recently, it was shown that the virus exists in an enveloped form (garnering membrane from the host to form the so-called quasi-enveloped HAV [eHAV] particle) while outside cells, potentially shielding itself from immune surveillance (Feng et al. 2013). This perhaps starts to blur the distinction between enveloped and unenveloped viruses and raises the question of whether this is unique to $\mathrm{HAV}$, or occurs also with some other picornaviruses, and has simply escaped attention. HAV also retains the ability to undergo transcytosis across cells when complexed with immunoglobulin A ( $\operatorname{IgA}$ ) antibody (Dotzauer et al. 2005; Counihan and Anderson 2016). HAV appears to have a very short (23 amino acids), nonmyristoylated VP4 (Shukla et al. 2014). Unlike other picornaviruses, on capsid assembly, a 67-residue polypeptide remains attached as a carboxy-terminal extension of VP1. By comparison with other picornaviruses, this would have been expected to belong to the nonstructural P2 section of the genome (protein $2 \mathrm{~A}$ in the other viruses); thus, it is known as VP12A or pX (see Pintó et al. 2018). pX is likely to mediate the association with the membrane envelope, and indeed is later cleaved off by host proteases to yield the mature, nonenveloped capsid (Feng et al. 2013). HAV isolates belong to a single serotype, and this lack of antigenic variation has been attributed to its need to conserve rare codons (Sanchez-Eugenia et al. 2016). These differences made the determination of its structure an imperative as it was expected that the capsid would bear distinguishing features to the structures from other genera and might shed light on its uncoating mechanism. Indeed, the analysis of HAV described below provided the first structural information on the Hepatovirus genus.

\section{OVERALL STRUCTURE OF HAV}

The structure was solved for HAV genotype TZ84, cultivated in 2BS cells and inactivated with formaldehyde by the Sinovac company
(Wang et al. 2015). Very thin, platelike crystals were grown for both the mature and natural empty virus (capsids that did not package RNA) and which were separated during sucrose density gradient purification. The crystals were grown in typical small drops from 30\% 2-methyl-2,4-pentanediol (MPD) and probably for this reason in situ data collection at room temperature (Axford et al. 2012) was impossible because when the x-ray shutter was opened the crystal would twitch out of the beam (see supplementary movie in Wang et al. 2015). Data were therefore collected from frozen crystals on beamlines I03 and I24 at Diamond Light Source, UK, and structures were solved for the mature and empty capsids at $3.5 \AA$ and $3.0 \AA$, respectively. Unlike some enteroviruses, such as enterovirus 71 (EV71) (Wang et al. 2012), the empty particles were essentially indistinguishable in exterior (and therefore antigenic) structure to the mature virus particle. As expected from previous experience with EV71 (Wang et al. 2012), there was no evidence for structural modification attributable to formaldehyde inactivation.

In terms of its broad surface architecture, the HAV particle most closely resembles the smooth, spherical aphthoviruses, although HAV has a noticeably faceted appearance, akin to a triakis icosahedron (Fig 1B). These flat facets mean that there is no canyon. Compared with a typical enterovirus (Rossmann et al. 1985), a shortening of the VP1 BC loop lowers the north wall of the canyon while reductions in the VP2 EF and VP1 GH loops ablate the south wall (Fig. 2A). Compared with the more spherical footand-mouth disease virus (FMDV) (Acharya et al. 1989), the loops at the fivefold and threefold axes in HAV are slightly raised to make the facets. There are no major depressions anywhere on the surface that might immediately suggest themselves as receptor attachment sites. The $\mathrm{HAV}$ receptor story remains incomplete; there was early evidence for the involvement of glycophorin A (Sanchez et al. 2004) as the erythrocyte receptor because HAV agglutinates red blood cells at acidic pHs. The epitope for glycophorin interactions was proposed to involve residue Lys 221 of VP1 and the surrounding VP3 residues (102-121) lining the "capsid pit." This is 
D.l. Stuart et al.

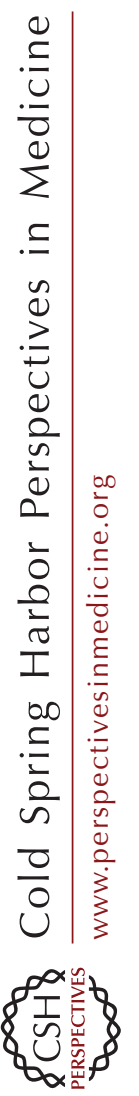

A

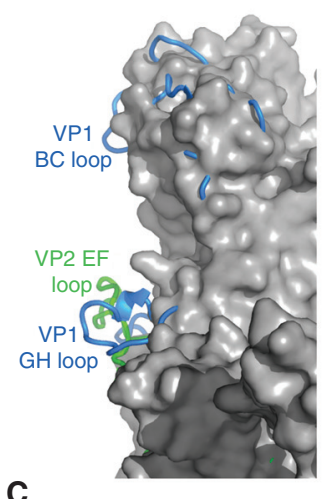

B
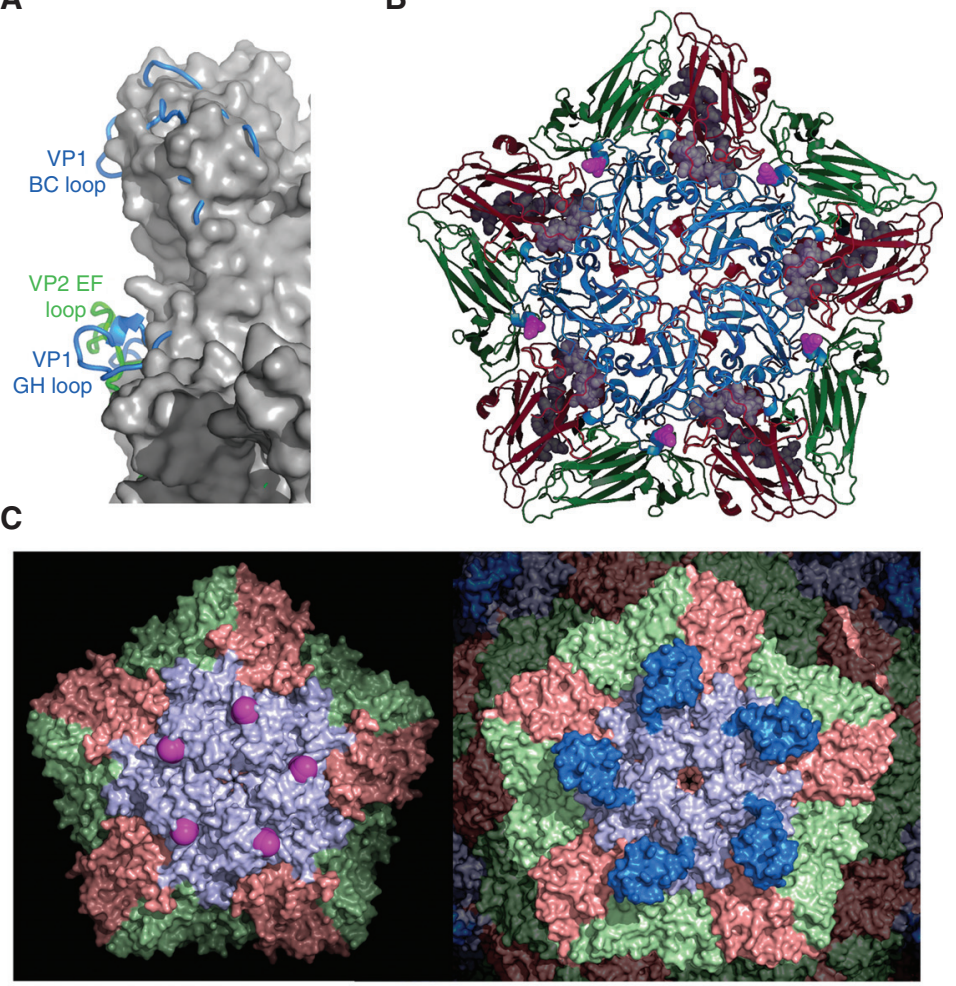

D
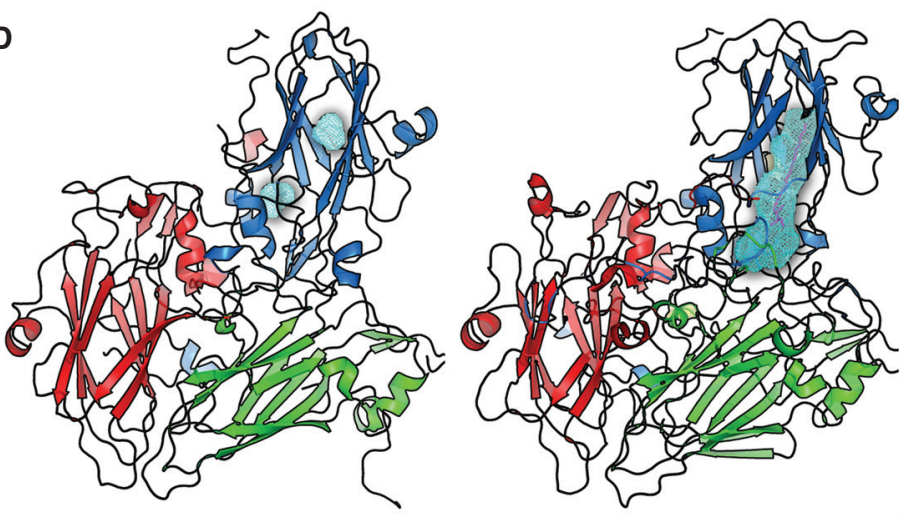

Figure 2. Distinctive structural features of hepatitis A virus (HAV). (A) No canyon depression. Side view of surface of protomer of HAV (gray) overlaid on a ribbon depiction of an enterovirus 71 (EV71) protomer with the proteins color-coded as in Figure 1B. This highlights the loops, which are labeled, that are truncated in HAV to flatten out the canyon. $(B)$ Potential glycophorin A receptor-binding site. A pentamer from the HAV capsid with viral protein (VP)1-3 drawn in cartoon representation showing the location of residues implicated in the glycophorin A receptor-binding site as spheres: magenta VP1 Lys 221 and gray 102-221 VP3. (C) VP1 extensions. Side-by-side surface renditions of an HAV pentamer (left) and a Ljungan virus (LV) pentamer (right). The magenta spheres highlight the last VP1 residue visible in HAV beyond which are $\sim 3$ VP1 residues and the $2 \mathrm{~A}$ extension that would be present in the enveloped particle. On the LV pentamer, the residues corresponding to the extended VP1 carboxy-terminus are drawn in royal blue. (D) Lack of hydrophobic pocket in VP1 $\beta$-barrel compared with an enterovirus. An HAV protomer (left) is drawn in cartoon representation alongside that for EV71 (right). The pocket volume calculated with PyMOL is drawn in light blue mesh. The lipid that binds in the EV71 pocket is drawn in magenta sticks. 
Hepatitis A Virus Capsid Structure

mapped to the structure in Figure 2B. Although there is no canyon or pit depression, the VP3 residues (102-121) do not appear to be accessible in the mature capsid. There have also been reports that T-cell immunoglobulin and mucin domain 1 (TIM1) acts as a receptor (Kaplan et al. 1996). However, recently, it has been shown to play no role in cellular entry for either quasienveloped or naked HAV, although it may facilitate early stages of infection by binding phosphatidylserine on the eHAV surface (Das et al. 2017). We believe the recently determined structure of a complex of HAV with a neutralizing antibody may throw some light on receptor binding, and this is discussed below.

In line with the low buoyant density in cesium chloride ( $\mathrm{CsCl}$ ) (Siegl et al. 1981), there are no apertures in the capsid to permit the entry of $\mathrm{Cs}^{+}$ions. In both the mature and empty capsids, approximately three residues are disordered at the carboxy-terminus of VP1 (where pX would previously have been joined to VP1). No extension is visible but its position on the outer capsid can be inferred. Interestingly, since the structure of HAV was determined, structures of other picornaviruses have been shown to bear an extension at the carboxy-terminus of VP1, which is more or less well-ordered on the surface of the capsid. An example of this is Ljungan virus (LV), in which $20 \AA$ high protrusions are seen, displaced some $45 \AA$ from the icosahedral fivefold axes, composed of the 55 carboxy-terminal residues (Fig. 2C) (Zhu et al. 2015).

Unlike enteroviruses (but in common with other picornavirus genera), HAV harbors no contiguous hydrophobic pocket in the VP1 $\beta$ barrel. The $\beta$-barrel is compressed compared with enteroviruses (Plevka et al. 2013; De Colibus et al. 2014), and the remaining space largely filled with hydrophobic side chains, as in aphtho- and cardioviruses (Fig. 2D) (Acharya et al. 1989). In addition, lengthened $\beta C$ and $\beta H$ strands in VP1 essentially cover what would be the entrance to the pocket. It therefore seems unlikely that HAV would be able to bind small molecules within this pocket as observed in enteroviruses and such a line of attack for antiviral compound discovery is unlikely to be fruitful (De Colibus et al. 2014).

\section{REWIRING OF THE PENTAMER BOUNDARY STRUCTURE}

One of the most significant differences between $\mathrm{HAV}$ and the earlier characterized mammalian picornavirus structures is a change in the structure at the icosahedral twofold axes. This comprises a swap in position for the amino-terminal 52 residues of VP2. The net result is that the overall structural appearance is almost indistinguishable because the same $\beta$-hairpin structure is formed at the twofold symmetric position; however, this hairpin comes from a different chain. This is achieved by a flip in the $\psi$ torsion angle of the peptide of residue 53, which lies very close to the twofold axis (only $\sim 5 \AA$ from it). This flip places the first 53 amino-acid residues across the interpentamer boundary (Fig. 3A), where they form a strong interaction with the adjacent pentamer. As a result of this swap in the amino-terminal structures, an extra strand is formed on the VP2 $\beta$-barrel of the neighboring pentamer. Similar structural domain swaps have been observed in certain protein structures (Liu and Eisenberg 2002). It results in a very similar overall structure in the case of HAV, but with complex changes in connectivity and in how the adjacent protomers of one pentamer are sewn together with the adjacent pentamer (Fig. 3A). This arrangement was not known to occur in picornaviruses when first recognized in HAV. However, it exists in distantly related picornalike viruses of insects including cricket paralysis virus (CrPV) (Tate et al. 1999), a member of the Cripavirus genus in the family Dicistroviridae. Furthermore, structures of several picornaviruses that harbor an uncleaved VP0 have since been solved, including LV (Zhu et al. 2015) and human parechovirus-1 (HPEV-1) (Kalynych et al. 2015), and have been found to have exactly the same VP0 swap (Fig. 3A). The domain-swapped amino-terminal conformation for VP2 is actually much closer to the amino-terminal conformation of VP3 and VP1. This further strengthens the suggestion that HAV belongs to a major clade of picornaviruses that are more "primitive" and are the most closely related to the insect picorna-like viruses such as CrPV (Tate et al. 1999) and triatoma virus (TrV) (Squires et al. 2013), also a cripavirus (Fig. 3B). 
D.l. Stuart et al.

A

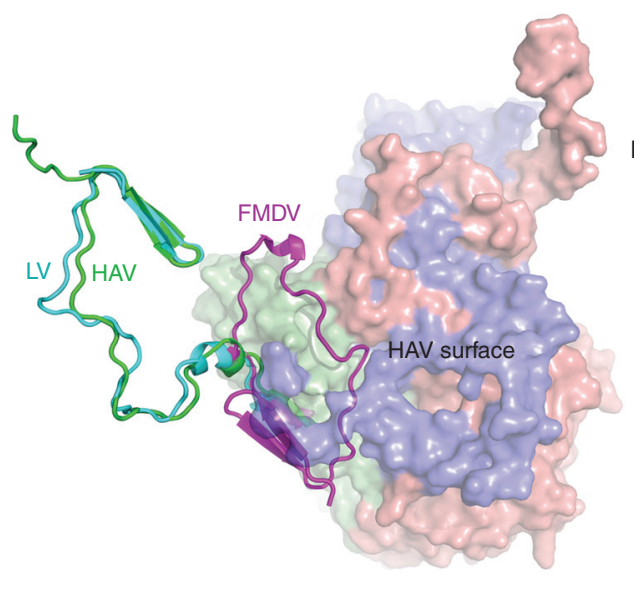

B

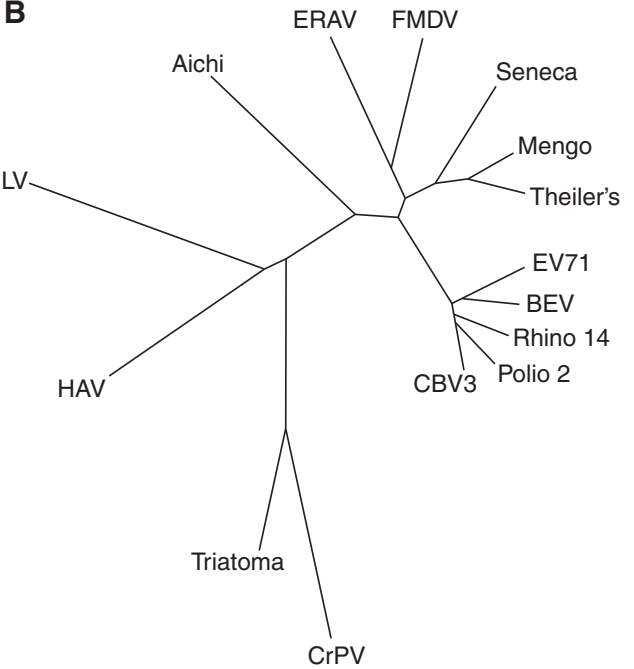

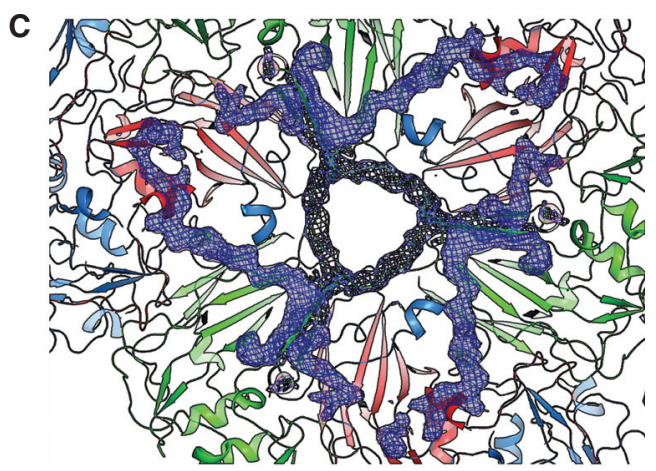

Figure 3. Structural features. (A) Viral protein (VP)2 domain swap. A surface-rendered protomer of hepatitis A virus (HAV) is depicted with the VP2 amino terminus differentiated by being drawn in green cartoon representation. The VP2 amino termini from Ljungan virus (LV) and foot-and-mouth disease virus (FMDV) are superimposed and depicted in cyan and magenta, respectively. It can be seen that both LV and HAV share the domain swap conformation characteristic of insect picorna-like viruses rather than the classical picornavirus conformation represented by FMDV. (B) Structure-based phylogenetic tree of representative picornaviruses and cripaviruses. Included in the analysis are 3VBF (EV71), 1BEV (bovine enterovirus), 4HRV (human rhinovirus14), 1EAH ( poliovirus type2), 1COV (coxsackievirus B3), 1TME (Theiler's virus), 3MEV (mengo virus), 3CJI (Seneca Valley virus), 1ZBA (FMDVA10), 2WFF (equine rhinitis A virus [ERAV]), 3NAP (triatoma virus [TrV]), 5GKA (aichi virus), 3JB4 (LV), and 1B35 (cricket paralysis virus [CrPV]). Evolutionary distance is calculated based on the number of unmatched residues and the deviation among matched residues. Residues corresponding to the HAV VP2 switch region (1-53) are excluded although their inclusion does not affect the result. $(C)$ Disordered region in the empty particle. HAV is viewed from the inside with blue mesh corresponding to positive $\left|F_{o}-F_{c}\right|$ electron density calculated taking the correctly positioned empty HAV from the full HAV. This shows that VP1 2-28 and VP2 5-17 around the threefold axis are better defined in the full particle.

\section{THE MYSTERY OF UNCOATING}

The extent of the structural differences detailed above strongly suggests that HAV uncoats via a mechanism distinct from enteroviruses. However, it also seems to be different from, for instance, the aphthoviruses, where there is a propensity for the capsid to fall apart into pentamers, whereas HAV is almost bulletproof. Does comparison of the structures of the mature virus and natural empty capsids throw any light on this? The two structures are almost identical external- 
ly, but internally the empty capsid has more disordered protein in the vicinity of the threefold axes (Fig. 3C), corresponding to regions of VP0 (the first 40 residues) and neighboring stretches of the amino terminus of VP1 (the first 47 residues). In the RNA-containing full particles, VP0 is at least partially cleaved and VP4 can be detected by sodium dodecyl sulfate polyacrylamide gel electrophoresis (SDS PAGE), although it is not visible in the structure (Wang et al. 2015). A greater proportion of VP0 appears to be uncleaved in HAV full particles than in other picornaviruses. This is consistent with previous suggestions that the VP0 cleavage is particularly slow (Probst et al. 1999). Empty HAV particles contain VP0 and appear to be similar to empty particles that are often observed in other picornavirus infections, where, in the absence of RNA, there is a tendency for the internal structure to be less well-ordered. It seems unlikely that such empty particles can encapsidate RNA and they are, therefore, probably not on the route to assembly of full particles.

In the full particles, residues 30-39 at the amino terminus of VP1 on the interior of the capsid are disordered. It is notable that this stretch is rich in lysine residues, which suggests that this region may interact with the RNA (the latter does not adhere to strict icosahedral symmetry and is not usually visible in structures where icosahedral averaging has been used to improve the phases). The use of a positively charged stretch of residues at the amino terminus of a major capsid protein has also been observed in LV and HPEVs, although in those cases the protein is VP3 rather than VP1 and the interaction with RNA is confirmed by the detailed visualization of sequence-specific interactions with packaging sequences (Zhu et al. 2015; Shakeel et al. 2017). Nevertheless, this suggests that this combination of charge interactions in addition to sequence-specific interactions may be a characteristic of this clade of picornaviruses. The sedimentation coefficients are circa 144S and $82 \mathrm{~S}$ for the full and natural empty particles, respectively (144S is a little less than the $155 \mathrm{~S}$ expected, for example, for a full enterovirus particle) (Wang et al. 2012). We have investigated the robustness of both empty and full particles, and both are extraordinarily tough compared with other picornaviruses (remaining stable at up to $80^{\circ} \mathrm{C}$ and at pHs down to about 2) (Walter et al. 2012). What is also unusual in HAV is that the empty capsids are extremely robust, whereas for most picornaviruses empty capsids and virus-like particles (VLPs) tend to be significantly less stable, posing a challenge to the design of VLP-based vaccines. It is possible that HAV may contain cryptic clues as to how to achieve increased stability in empty particles.

In many picornaviruses, the stability of the particle is determined by the interface separating the 12 pentameric assemblies comprising the icosahedral capsid (Filman et al. 1989; Warwicker 1992; Porta et al. 2013). To determine whether the stability of HAV might arise from the VP2 domain swap at the pentamer interface, we characterized CrPV capsid stability because it shares the domain swap seen in HAV. We found that the stability profile of $\mathrm{CrPV}$ was similar to the enteroviruses. Therefore, the VP2 domain swap cannot be explained as a strategy to achieve the high physical stability of the HAV capsid.

In the HAV capsid, however, there is particularly tight packing in the interaction region adjacent to the twofold axes that separate in the initial stages of uncoating in the enteroviruses (Garriga et al. 2012; Wang et al. 2012). The complementarity in HAV resembles that seen in poliovirus, where the release of pocket factor likely triggers instability. This complimentarity is achieved by matching tyrosine side chains with small residues nestled around the twofold axis. This could contribute significantly to high HAV particle stability, while the VP2 domain swap could be indicative of a fundamental difference in how HAV uncoats its genome compared with enteroviruses. Apart from these two striking features, the other candidate interface, relating to protomers around the icosahedral fivefold axes, has similar properties to other picornaviruses.

Like enteroviruses, HAV could transfer its genome across the endosomal membrane to the cytoplasm using a mechanism involving the formation of an "umbilicus"-like structure comprised of the amphipathic amino-terminal helix 
D.l. Stuart et al.

of VP1 and a membrane pore generated by VP4 (Ren et al. 2013; Butan et al. 2014). The shortened, non-myristoylated version of VP4 in HAV has some hydrophobic character consistent with the formation of an amphipathic helix, and it has been reported to rupture membranes by formation of discrete pores (Shukla et al. 2014) as has the VP4 from TrV (Sanchez-Eugenia et al. 2015). Although HAV is capable of undergoing transcytosis when complexed with an antibody (Dotzauer et al. 2000, 2005; Counihan and Anderson 2016), HAV transcytosis is inefficient and likely involves vesicular transport, not entry of intact HAV particles across the plasma or endosomal membrane directly into the cytoplasm. It is not clear what mechanism if any could provide for entry of the capsid into the cytosol, or what specific host factor (perhaps a protease) could be capable of triggering particle disassembly within the cytoplasm. Thus, despite the seemingly unsurmountable challenge posed by the stability of the HAV capsid, uncoating is likely to follow interactions with a receptor expressed on membranes, as in the enteroviruses.

The interface between the pentameric assemblies in the HAV capsid is strengthened by a combination of factors: the domain swap crossover and the supplementation of $\beta$-sheets that span the twofold axis, the enhanced complementarity of the helices at the twofold axis, and the overall tighter knit of the capsid (which has no holes, as mentioned above) compared with other picornaviruses. This makes it quite mysterious how the particle disassembles. By reference to the Dicistroviridae, the structure of empty, heated, $\operatorname{TrV}$ particles revealed that the capsids were not expanded nor had any channels by which the RNA could have exited contrasting to the enterovirus-heated particles. Internally, the first 33 residues of VP1 were disordered but more significantly, the first 76 residues of VP2 comprising the entire domain swap portion were also disordered. It was suggested that RNA release may take place through transient flipping out of pentamer subunits (Sanchez-Eugenia et al. 2016). Although there is no direct evidence to support this model, it seems likely that TrV and HAV share a common mechanism, and structural analysis, most likely by cryoelectron microscopy (cryo-EM), of transient or trapped intermediates should be able to resolve this puzzle.

\section{QUASI-ENVELOPED EHAVAND ENDOSOMAL SORTING COMPLEX REQUIRED FOR TRANSPORT (ESCRT) ENGAGEMENT REMAINS A STRUCTURAL PUZZLE}

As noted above, HAV is the first picornavirus for which nonlytic cellular egress in extracellular vesicles has been found likely to be important in disease pathogenesis. How this happens remains obscure, and the origins of the surrounding membranes have also been poorly understood. Recently, however, McKnight et al. (2017) reported a quantitative proteomics analysis of eHAV virions, which showed that capsids are selected as cargo for vesicular export via a highly specific process, and that infectious eHAV virions possess a set of host proteins similar to that of exosomes, enriched in endolysosomal components and missing markers of autophagy, suggesting an exosome-like mechanism of ESCRT-mediated eHAV biogenesis involving endosomal budding. Note that, as for many other aspects of $\mathrm{HAV}$, this is quite different from the autophagosome-mediated release proposed for enteroviruses. Further studies showed that ESCRT-III proteins, particularly charged multivesicular body protein 2A (CHMP2A), have a role in eHAV biogenesis, as previously proposed (McKnight et al. 2017). This is in line with the observation (based on knockdown and mutagenesis studies) that HAV harbors tandem candidate $\mathrm{YPX}_{3} \mathrm{~L}$ motifs (VP2: $\mathrm{Y}_{144} \mathrm{PHGLL}_{149}$ and $\mathrm{Y}_{177} \mathrm{PVWEL}_{182}$ ), that putatively bind a component of the ESCRT pathway, ALG-2-interacting protein X (ALIX) (Feng et al. 2013). This would then allow the virus to engage the ESCRT complex to facilitate release of enveloped particles via exocytosis (Feng et al. 2013). However, as shown in Figure 4, the residues implicated are surprisingly mostly buried, apart from E181 and P178, making their role unclear unless major conformational rearrangements render them accessible, or if they are accessible on a precursor particle. Structural analysis of eHAV particles might clarify this. 


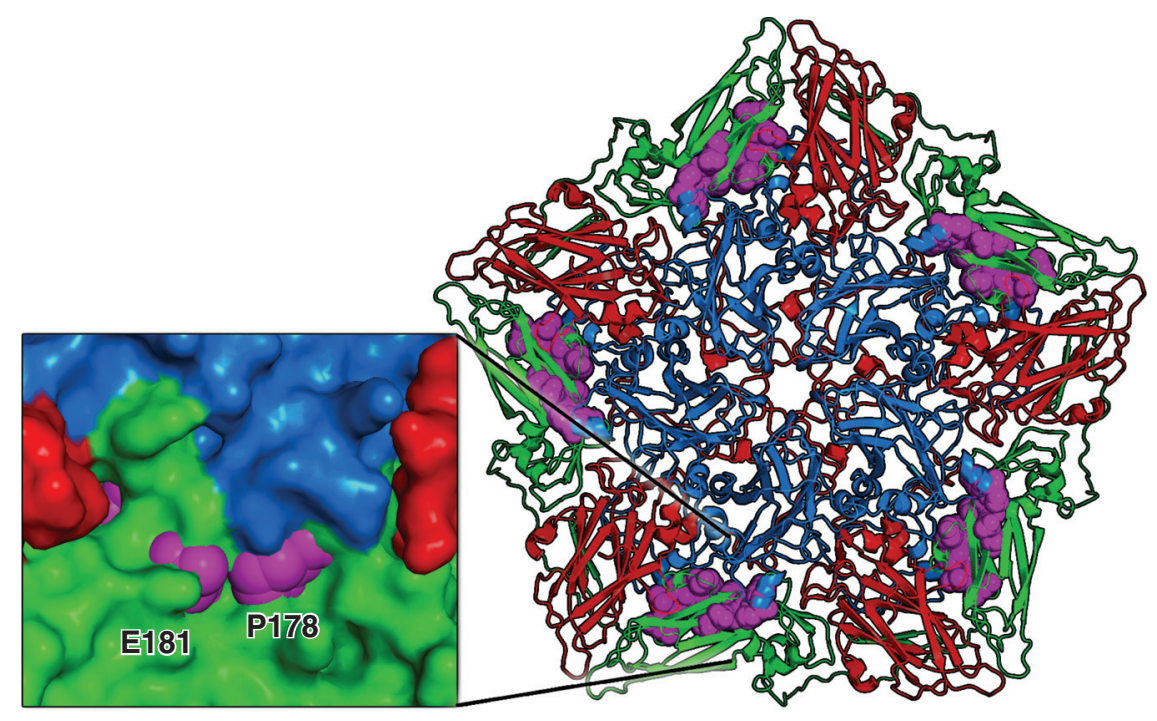

Figure 4. Location of endosomal sorting complex required for transport (ESCRT) late-domain motifs. Latedomain $\mathrm{YPX}_{3} \mathrm{~L}$ motifs are shown as magenta spheres on the pentamer of hepatitis A virus (as drawn in Fig. 2A). In the inset box, a close-up of the residues that are accessible is shown in the context of the accessible surface.

\section{DOES THE ANTIGENIC SURFACE OF HAV COMPRISE ESSENTIALLY A SINGLE ANTIGENIC SITE?}

Several studies have addressed the antigenic structure of the HAV capsid, largely built around the characterization of a number of neutralizing monoclonal antibodies (mAbs). Until recently, the evidence for residues comprising part of the binding sites of such antibodies was indirect, mainly that viruses harboring mutations at a certain point escaped neutralization. On this basis, residues S102, V171, A176, and K221 of VP1 and D70, S71, Q74, and 102-121 of VP3 were implicated in neutralizing epitopes (Ping and Lemon 1992). All except K221 of VP1 were proposed to form part of a single antigenic site; but since these residues are separated by $40-50 \AA$ on the particle surface, they cannot form part of any single epitope. In a more recent study, $12 \mathrm{mAbs}$ were generated against HAV particles (Wang et al. 2015), one of which, $\mathrm{mAb} \# 11$, was found to have both neutralizing activity as well as the ability to bind VP3 in immunoblots, providing evidence of direct physical interaction with VP3. Based on the particle structure, peptides representing all exposed regions on the surface of the capsid were synthesized. When tested for their ability to be bound by $\mathrm{mAb} \# 11$, peptides representing VP3 residues 67-77 and 208-219 reacted mildly. These VP3 peptide sequences are relatively closely positioned on the surface of the virus, and thus may contribute to an augmented version of the major conformational antigenic site identified by Ping and Lemon (1992) previously.

To identify additional epitopes on the surface of HAV, structure-based predictive methodology (Borley et al. 2013) was applied. The results concurred with those above and suggested that VP2, residues 71 and 198, and VP3 residues 89-96 may comprise additional epitopes. More recently direct evidence has come from a high-resolution cryo-EM complex between HAV and a potent HAV-specific mAb, R10 (Wang et al. 2017). This antibody is an extremely potent neutralizer (neut $_{50}$ is $\sim 2 \mathrm{nM}, \sim 0.3 \mu \mathrm{g} / \mathrm{mL}$ for the antibody and $\sim 3 \mathrm{nM}, \sim 0.45 \mu \mathrm{g} / \mathrm{mL}$ for the antigen-binding fragment $[\mathrm{Fab}]$ ) and reverse transcription polymerase chain reaction (RT-PCR) experiments to quantify virus remaining on the cell surface showed that not only could the antibody block attachment but it could actively compete with the receptors on the cell surface (Wang et al. 


\section{D.I. Stuart et al.}

2017). These experiments show that R10 neutralizes HAV infection by blocking attachment to the host cell. The complex thus reveals not only atomic details of the antibody-binding site (Fig. 5), but also suggests where cell attachment might occur. Despite binding being extremely tight, only the
Fab fragment destabilizes the capsid, and then only relatively mildly (reducing the melting temperature by $7^{\circ} \mathrm{C}$ ), whereas the antibody slightly stabilizes the particle (by $2^{\circ} \mathrm{C}$ ), consistent with bivalent attachment cross-linking adjacent pentameric building blocks (Wang et al. 2017).

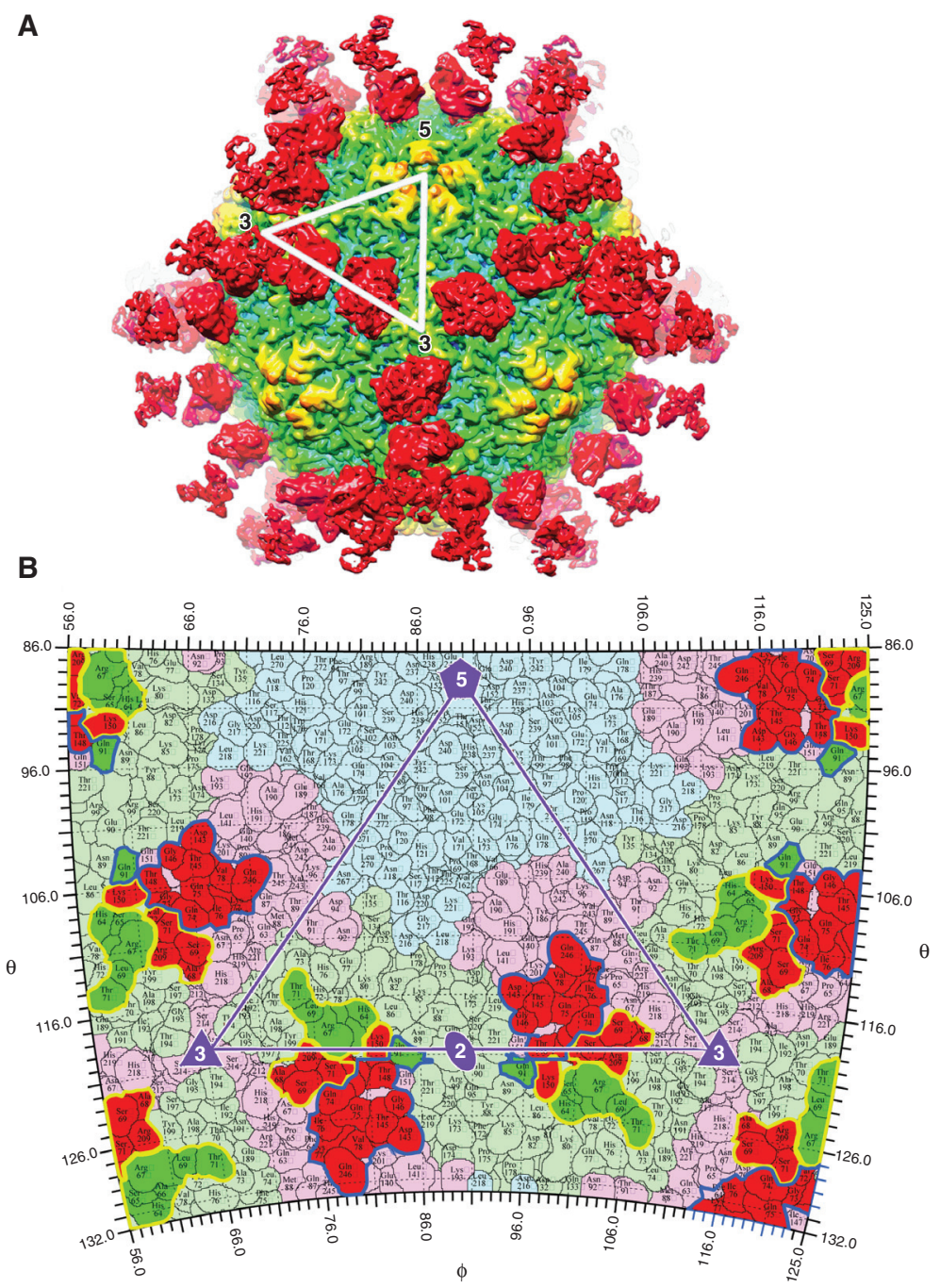

Figure 5. R10 antibody interactions with hepatitis A virus (HAV). (A) Surface of the cryoelectron microscopy (cryo-EM) complex for R10 and HAV based on the $4.2 \AA$ resolution cryo-EM structure of the R10 antigenbinding fragment (Fab)/HAV full particle complex. The virus surface is color-coded blue to red according to distance from the particle center while the Fab molecules are shown in red. One icosahedral asymmetric unit is indicated by a white triangle. $(B)$ Surface maps of HAV generated using radial interpretation of viral electron density maps (RIVEM) (Chapman and Rossmann 1993). Residues of VP1, VP2, and VP3 are shaded in pale blue, green, and red, respectively; residues involved in binding to R10 are shown in brighter colors corresponding to the protein chain they belong to, and are outlined by blue and yellow lines. An icosahedral protomer is outlined in purple with the symmetry axes labeled. (From Wang et al. 2017; panel reproduced $(A)$ and modified $(B)$, respectively, with permission from the author.) 
Hepatitis A Virus Capsid Structure

The details of the R10 epitope are shown in Figure 5B. The heavy chain predominantly binds VP3 and contributes $\sim 60 \%$ of the protein-protein interface, whereas the light chain binds VP2 and VP3 and contributes $\sim 40 \%$. The interaction surface comprises four of the six common R10 complementary determining regions (CDRs): H1 (residues 28-32), H2 (residues 52-57), H3 (residues 100-106), and L1 (residues 30-31). Atypically, the light-chain framework region (L1-2, residues 45-55) provides for additional interactions. VP2 residues $64-71$ and VP3 residues $68-78,143-150,209$, and 246 form the epitope on the surface of the capsid. Altogether, these residues are $87.5 \%$ identical and $91.7 \%$ conserved across the six primate hepatovirus genotypes (see McKnight and Lemon 2018), suggesting that R10 probably binds strongly to most if not all of these viruses. The affinity of R10 for the capsid is strengthened by 19 hydrogen bonds, which are formed in part by the side chain of Y48 in the light chain, and main chain carbonyl groups from V103 (H-chain) and L53 (L-chain). These interact with VP3 K150 and R209, respectively, and indirectly with VP2 R67. In addition, unpublished results (XW) show that a further four neutralizing antibodies attach at essentially the same epitope. So how do these compelling structural results stack up against the previous serological results? In brief, a subset of the residues previously mapped as being part of the epitope are confirmed, as is even the proposal, from in silico analysis, that VP2 might be part of the epitope. However, there is absolutely no possibility of any of the VP1 residues directly influencing binding of this antibody. We will return to this puzzle after considering what these results might tell us about receptor binding.

All the above results are in line with neutralization being achieved through blocking of receptor attachment. This pinpoints the pentamer interface as the site for receptor recognition. Surprisingly, the crystal structure revealed that the surface of the virus shows a marked segregation of charge: broadly, the pentamers are edged by a positively charged fringe, with the central region being mainly negatively charged. The positive charges on the fringe are decorated with a string of sulfate ions derived from the crystallization media. In addition, there are 10 sulfates grouped close to each icosahedral fivefold axis. This might suggest that sulfated glycosaminoglycans may act as, or form part of, the receptor and are supported by competition assays in which the virus is preincubated with heparin sulfate, which shows marked inhibition at micromolar concentration (Wang et al. 2017). These results, together with our observation that the R10 Fab destabilizes the capsid, suggest the use of a receptor mimic mechanism to neutralize virus infection, suggesting new opportunities for therapeutic intervention.

We are still left with the puzzle of HAV apparently possessing a single, hugely extended antigenic site. There are examples for other viruses where mutations resulting in altered antigenic properties are not always directly involved in binding antibodies (Parry et al. 1990). Now, because the VP1 residues are only implicated by escape mutation data, we propose that the predominate neutralizing epitope is that visualized by Wang et al. (2017), and that the escape observed via changes in VP1 is achieved by some other mechanism. The mechanism remains unknown, but because the virus is very rigid, and many of these neutralizing antibodies are quite potent binders, perhaps the most likely explanation is that escape is achieved by circumventing the block on receptor binding for instance by relaxing the need for receptor attachment to the VP2/3 epitope area, conceivably by boosting receptor attachment (perhaps an alternative receptor) to VP1. In this respect, we note that one of the escape mutations (VP1 221) occurs at a residue also implicated in glycophorin attachment, while another (VP1 102) is adjacent to a sulfate-binding site, with two others (VP1 171 and 176) being relatively close by.

\section{CONCLUDING REMARKS}

There are subtle but profound differences in the structure of the HAV capsid compared with that of previously characterized picornaviruses. A structure-based phylogeny (Fig. 3B) suggests that HAV may represent a link between "classical" mammalian picornaviruses and more primitive picorna-like viruses that infect insects. The 
D.I. Stuart et al.

striking amino-terminal domain swap in VP2 renders its structure more similar to the homologous VP1 and VP3 proteins, providing support for the concept that HAV retains structural and functional features characteristic of primordial picornavirus ancestors, which bore stronger hallmarks of their $T=3$ origins. Such archaic viruses may have been more akin to presentday insect picorna-like viruses, and the enormous diversity that characterizes present-day picornaviruses may have been generated by evolutionary pressure to evolve efficient mechanisms of cell entry. Further research into this virus, in particular structural analyses of the enveloped particles, as well as the capture of assembly states and attachment/uncoating intermediates, is expected to explain more of its unique biology and bridge gaps in our knowledge of the virosphere.

\section{ACKNOWLEDGMENTS}

This work is supported by the National Major Project of Infectious Disease, the Ministry of Science and Technology 973 Project (Grant No. 2014CB542800), National Science Foundation (Grant No. 81330036), and the Strategic Priority Research Program of the Chinese Academy of Sciences (Grant No. XDB08020200). D.I.S. and E.E.F. are supported by the UK Medical Research Council (Grant Nos. G1000099 and MR/N00065X/1) and J.R. by the Wellcome Trust.

\section{REFERENCES}

${ }^{*}$ Reference is also in this collection.

Acharya R, Fry E, Stuart D, Fox G, Rowlands D, Brown F. 1989. The three-dimensional structure of foot-andmouth disease virus at $2.9 \AA$ resolution. Nature 337: 709-716.

Axford D, Owen RL, Aishima J, Foadi J, Morgan AW, Robinson JI, Nettleship JE, Owens RJ, Moraes I, Fry EE, et al 2012. In situ macromolecular crystallography using microbeams. Acta Crystallogr D Biol Crystallogr 68: 592600.

Borley DW, Mahapatra M, Paton DJ, Esnouf RM, Stuart DI, Fry EE. 2013. Evaluation and use of in-silico structurebased epitope prediction with foot-and-mouth disease virus. PloS ONE 8: e61122.
Butan C, Filman DJ, Hogle JM. 2014. Cryo-electron microscopy reconstruction shows poliovirus $135 \mathrm{~S}$ particles poised for membrane interaction and RNA release. J Virol 88: $1758-1770$.

Chapman MS, Rossmann MG. 1993. Comparison of surface properties of picornaviruses: Strategies for hiding the receptor site from immune surveillance. Virology 195: 745756.

Counihan NA, Anderson DA. 2016. Specific IgA enhances the transcytosis and excretion of hepatitis A virus. Sci Rep 6: 21855 .

Das A, Hirai-Yuki A, Gonzalez-Lopez O, Rhein B, MollerTank S, Brouillette R, Hensley L, Misumi I, Lovell W, Cullen JM, et al. 2017. TIM1 (HAVCR1) is not essential for cellular entry of either quasi-enveloped or naked hepatitis A virions. $m$ Bio 8: $\mathrm{e} 00969$.

De Colibus L, Wang X, Spyrou JA, Kelly J, Ren J, Grimes J, Puerstinger G, Stonehouse N, Walter TS, Hu Z, et al. 2014. More-powerful virus inhibitors from structurebased analysis of HEV71 capsid-binding molecules. Nat Struct Mol Biol 21: 282-288.

Dotzauer A, Gebhardt U, Bieback K, Gottke U, Kracke A, Mages J, Lemon SM, Vallbracht A. 2000. Hepatitis A virus-specific immunoglobulin A mediates infection of hepatocytes with hepatitis A virus via the asialoglycoprotein receptor. J Virol 74: 10950-10957.

Dotzauer A, Brenner M, Gebhardt U, Vallbracht A. 2005. IgA-coated particles of Hepatitis A virus are translocalized antivectorially from the apical to the basolateral site of polarized epithelial cells via the polymeric immunoglobulin receptor. J Gen Virol 86: 2747-2751.

Feng Z, Hensley L, McKnight KL, Hu F, Madden V, Ping L, Jeong SH, Walker C, Lanford RE, Lemon SM. 2013. A pathogenic picornavirus acquires an envelope by hijacking cellular membranes. Nature 496: 367-371.

Filman DJ, Syed R, Chow M, Macadam AJ, Minor PD, Hogle JM. 1989. Structural factors that control conformational transitions and serotype specificity in type 3 poliovirus. EMBO J 8: 1567-1579.

Garriga D, Pickl-Herk A, Luque D, Wruss J, Caston JR, Blaas D, Verdaguer N. 2012. Insights into minor group rhinovirus uncoating: The X-ray structure of the HRV2 empty capsid. PLoS Pathog 8: e1002473.

Kalynych S, Palkova L, Plevka P. 2015. The structure of human parechovirus 1 reveals an association of the RNA genome with the capsid. J Virol 90: 1377-1386.

Kaplan G, Totsuka A, Thompson P, Akatsuka T, Moritsugu Y, Feinstone SM. 1996. Identification of a surface glycoprotein on African green monkey kidney cells as a receptor for hepatitis A virus. EMBO J 15: 4282-4296.

Liu Y, Eisenberg D. 2002. 3D domain swapping: As domains continue to swap. Protein Sci 11: 1285-1299.

* McKnight KL, Lemon SM. 2018. Hepatitis A virus genome organization and replication strategy. Cold Spring Harb Perspect Med doi: 10.1101/cshperspect.a033480.

McKnight KL, Xie L, Gonzalez-Lopez O, Rivera-Serrano EE, Chen X, Lemon SM. 2017. Protein composition of the hepatitis A virus quasi-envelope. Proc Natl Acad Sci 114: 6587-6592.

Parry N, Fox G, Rowlands D, Brown F, Fry E, Acharya R, Logan D, Stuart D. 1990. Structural and serological evi- 
dence for a novel mechanism of antigenic variation in foot-and-mouth disease virus. Nature 347: 569-572.

Ping LH, Lemon SM. 1992. Antigenic structure of human hepatitis A virus defined by analysis of escape mutants selected against murine monoclonal antibodies. J Virol 66: $2208-2216$.

* Pintó RM, Pérez-Rodríguez F-J, D’Andrea L, de Castellarnau M, Guix S, Bosch A. 2018. HAV codon usage: Implications for translation kinetics and capsid folding. Cold Spring Harb Perspect Med doi: 10.1101/cshperspect. a031781.

Plevka P, Perera R, Yap ML, Cardosa J, Kuhn RJ, Rossmann MG. 2013. Structure of human enterovirus 71 in complex with a capsid-binding inhibitor. Proc Natl Acad Sci 110: 5463-5467.

Porta C, Kotecha A, Burman A, Jackson T, Ren J, Loureiro S, Jones IM, Fry EE, Stuart DI, Charleston B. 2013. Rational engineering of recombinant picornavirus capsids to produce safe, protective vaccine antigen. PLoS Path 9: e1003255.

Probst C, Jecht M, Gauss-Muller V. 1999. Intrinsic signals for the assembly of hepatitis A virus particles. Role of structural proteins VP4 and 2A.J Biol Chem 274: 45274531.

Ren J, Wang X, Hu Z, Gao Q, Sun Y, Li X, Porta C, Walter TS, Gilbert RJ, Zhao Y, et al. 2013. Picornavirus uncoating intermediate captured in atomic detail. Nat Commun 4: 1929.

Rossmann MG, Johnson JE. 1989. Icosahedral RNA virus structure. Annu Rev Biochem 58: 533-573.

Rossmann MG, Arnold E, Erickson JW, Frankenberger EA, Griffith JP, Hecht HJ, Johnson JE, Kamer G, Luo M, Mosser AG, et al. 1985. Structure of a human common cold virus and functional relationship to other picornaviruses. Nature 317: 145-153.

Sanchez G, Aragones L, Costafreda MI, Ribes E, Bosch A Pinto RM. 2004. Capsid region involved in hepatitis A virus binding to glycophorin A of the erythrocyte membrane. J Virol 78: 9807-9813.

Sanchez-Eugenia R, Goikolea J, Gil-Carton D, Sanchez-Magraner L, Guerin DM. 2015. Triatoma virus recombinant VP4 protein induces membrane permeability through dynamic pores. J Virol 89: 4645-4654.

Sanchez-Eugenia R, Durana A, Lopez-Marijuan I, Marti GA, Guerin DM. 2016. X-ray structure of Triatoma virus empty capsid: Insights into the mechanism of uncoating and RNA release in dicistroviruses. J Gen Virol 97: 2769-2779.
Shakeel S, Dykeman EC, White SJ, Ora A, Cockburn JJB, Butcher SJ, Stockley PG, Twarock R. 2017. Genomic RNA folding mediates assembly of human parechovirus. Nat Communs 8: 5.

Shukla A, Padhi AK, Gomes J, Banerjee M. 2014. The VP4 peptide of hepatitis A virus ruptures membranes through formation of discrete pores. J Virol 88: 12409-12421.

Siegl G, Frosner GG, Gauss-Muller V, Tratschin JD, Deinhardt F. 1981. The physicochemical properties of infectious hepatitis A virions. J Gen Virol 57: 331-341.

Smith TJ, Kremer MJ, Luo M, Vriend G, Arnold E, Kamer G, Rossmann MG, McKinlay MA, Diana GD, Otto MJ. 1986. The site of attachment in human rhinovirus 14 for antiviral agents that inhibit uncoating. Science 233: 12861293.

Squires G, Pous J, Agirre J, Rozas-Dennis GS, Costabel MD, Marti GA, Navaza J, Bressanelli S, Guerin DM, Rey FA. 2013. Structure of the Triatoma virus capsid. Acta Crystallogr D Biol Crystallogr 69: 1026-1037.

Tate J, Liljas L, Scotti P, Christian P, Lin T, Johnson JE. 1999. The crystal structure of cricket paralysis virus: The first view of a new virus family. Nat Struct Biol 6: 765-774.

Walter TS, Ren J, Tuthill TJ, Rowlands DJ, Stuart DI, Fry EE. 2012. A plate-based high-throughput assay for virus stability and vaccine formulation. J Virol Methods $\mathbf{1 8 5}$ 166-170.

Wang X, Peng W, Ren J, Hu Z, Xu J, Lou Z, Li X, Yin W, Shen X, Porta C, et al. 2012. A sensor-adaptor mechanism for enterovirus uncoating from structures of EV71. Nat Struct Mol Biol 19: 424-429.

Wang X, Ren J, Gao Q, Hu Z, Sun Y, Li X, Rowlands DJ, Yin W, Wang J, Stuart DI, et al. 2015. Hepatitis A virus and the origins of picornaviruses. Nature 517: 85-88.

Wang X, Zhu L, Dang M, Hu Z, Gao Q, Yuan S, Sun Y, Zhang B, Ren J, Kotecha A, et al. 2017. Potent neutralization of hepatitis A virus reveals a receptor mimic mechanism and the receptor recognition site. Proc Natl Acad Sci 114: 770775.

Warwicker J. 1992. Model for the differential stabilities of rhinovirus and poliovirus to mild acidic $\mathrm{pH}$, based on electrostatics calculations. J Mol Biol 223: 247-257.

Zhu L, Wang X, Ren J, Porta C, Wenham H, Ekstrom JO, Panjwani A, Knowles NJ, Kotecha A, Siebert CA, et al. 2015. Structure of Ljungan virus provides insight into genome packaging of this picornavirus. Nat Commun $\mathbf{6}$ : 8316. 


\section{$\&_{\mathrm{CSH}}^{\infty} \&$ Cold Spring Harbor

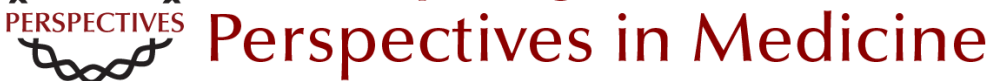

\section{Hepatitis A Virus Capsid Structure}

David I. Stuart, Jingshan Ren, Xiangxi Wang, Zihe Rao and Elizabeth E. Fry

Cold Spring Harb Perspect Med 2019; doi: 10.1101/cshperspect.a031807 originally published online July 23, 2018

\section{Subject Collection Enteric Hepatitis Viruses}

Hepatitis A Virus Genome Organization and Replication Strategy

Kevin L. McKnight and Stanley M. Lemon

Adaptive Immune Responses in Hepatitis A Virus and Hepatitis E Virus Infections

Christopher M. Walker

Small Animal Models of Hepatitis E Virus Infection Tian-Cheng Li and Takaji Wakita

Acute and Persistent Hepatitis E Virus Genotype 3 and 4 Infection: Clinical Features, Pathogenesis, and Treatment Nassim Kamar and Sven Pischke

Epidemiology of Genotype 1 and 2 Hepatitis E Virus Infections

Kenrad E. Nelson, Alain B. Labrique and Brittany L. Kmush

History of the Discovery of Hepatitis A Virus Stephen M. Feinstone

Epidemiology and Transmission of Hepatitis A Virus and Hepatitis E Virus Infections in the United States

Megan G. Hofmeister, Monique A. Foster and Eyasu H. Teshale

Stem Cell-Derived Culture Models of Hepatitis E Virus Infection

Viet Loan Dao Thi, Xianfang Wu and Charles M. Rice
Evolutionary Origins of Enteric Hepatitis Viruses Anna-Lena Sander, Victor Max Corman, Alexander N. Lukashev, et al.

Enterically Transmitted Non-A, Non-B Hepatitis and the Discovery of Hepatitis $\mathrm{E}$ Virus

Stanley M. Lemon and Christopher M. Walker

Natural History, Clinical Manifestations, and

Pathogenesis of Hepatitis E Virus Genotype 1 and

2 Infections

Rakesh Aggarwal and Amit Goel

Hepatitis A Virus and Hepatitis E Virus: Emerging and Re-Emerging Enterically Transmitted

Hepatitis Viruses

Stanley M. Lemon and Christopher M. Walker

Hepatitis A Virus Capsid Structure

David I. Stuart, Jingshan Ren, Xiangxi Wang, et al.

Comparative Pathology of Hepatitis A Virus and Hepatitis E Virus Infection John M. Cullen and Stanley M. Lemon

Innate Immunity to Enteric Hepatitis Viruses Zongdi Feng and Stanley M. Lemon

Nonhuman Primate Models of Hepatitis A Virus and Hepatitis E Virus Infections

Robert E. Lanford, Christopher M. Walker and

Stanley M. Lemon

For additional articles in this collection, see http://perspectivesinmedicine.cshlp.org/cgi/collection/ 